\title{
CHANGES IN EXPRESSION OF GLIAL ANTIGENS M1 AND C1 AFTER CEREBELLAR INJURY ${ }^{1}$
}

\author{
CARL LAGENAUR, COLIN MASTERS, AND MELITTA SCHACHNER ${ }^{2}$ \\ Department of Neurobiology, University of Heidelberg, Im Neuenheimer Feld 504, D-6900 Heidelberg, \\ Federal Republic of Germany
}

Received August 10, 1981; Revised November 18, 1981; Accepted November 24, 1981

\begin{abstract}
In response to mechanical injury in the adult mouse cerebellum, Bergmann glia and astrocytes of the granular layer exhibit abnormally increased expression of $\mathrm{MI}$ antigen, while expression of $\mathrm{Cl}$ antigen in Bergmann glia is reduced. These reciprocal changes in two different astrocytic antigenic determinants (cach recognized by monoclonal antibodies) are casily detected in the immediate area of the wound 4 days after the lesion. Although loss of $\mathrm{C} 1$ antigen from Bergmann glia remains localized to the area of the wound, abnormal M1 expression becomes widespread in cerebellar astrocytes, also affecting the contralateral side of the injured cerebellum at its peak 8 to 12 days after injury.

These findings suggest that previous observations of abnormal expression of the two antigens in the cerebellum of mutant mice (Sommer, I., and M. Schachner (1981) J. Supramol. Struct. 16: 5374) might be interpreted with the view that a glial reaction to a pathological state might be induced by genetically programmed neuronal cell death and/or abnormal development. We therefore postulate that expression of M1 in astrocytes that normally do not express this antigen and repression of $\mathrm{C} 1$ in normally positive astrocytes are indicative of a distinct functional state of astroglia, reactive gliosis.
\end{abstract}

Two monoclonal antibodies, $\mathrm{M} 1$ and $\mathrm{C} 1$, recognize different subclasses of astrocytes in the adult mouse nervous system (Lagenaur et al., 1980; Sommer et al., 1981). In the adult cerebellar cortex, M1 antigen is expressed predominantly in astrocytes of the white matter but is not detectable by immunofluorescence in astrocytes either of the granular layer or of the molecular layer (Bergmann glial cells) (Lagenaur et al., 1980). In contrast, $\mathrm{C} 1$ antigen is localized in adult Bergmann glia but not in astrocytes of the granular layer or white matter (Sommer et al., 1981). Both M1 and C1 have intracellular distributions in astroglia that are similar to the distribution of the intermediate filament component, glial fibrillary acidic protein. This similarity is particularly prominent in cultured astroglia in which filament bundles are easily discernible (Lagenaur et al., 1980, Sommer et al., 1981). In four distinct mutations affecting the mouse cerebellum (reeler, staggerer, weaver, and Purkinje cell

\footnotetext{
${ }^{1}$ We are indebted to Drs. A. Bignami, D. Dahl, and L. F. Eng for gifts of antiserum to glial fibrillary acidic protein. This work was supported by Deutsche Forschungsgemeinschaft (Scha 185/b), a National Institutes of Health fellowship (Nr. IF32NS06058-01) to C. L., and an Alexander von Humboldt fellowship to C. M.

${ }^{2}$ To whom correspondence should be addressed.
}

degeneration), M1 is expressed abnormally in Bergmann glia and in astrocytes in the granular layer, while $\mathrm{Cl}$ antigen progressively disappears from Bergmann glia in the mutants (Lagenaur et al., 1980; Sommer et al., 1981; Sommer and Schachner, 1981). Since these mutations involve distinct cellular abnormalities and occur at different gene loci, the common feature underlying the abnormal antigenic expression of $\mathrm{M} 1$ and $\mathrm{C} 1$ antigens was postulated to result from a more general pathological reaction of astrocytes in mutant cerebella.

The present study was performed to investigate the expression of $\mathrm{M} 1$ and $\mathrm{C} 1$ antigens in astrocytes reacting to a localized injury in which both normal resting and reactive astrocytes could be expected to be present within one cerebellum.

\section{Materials and Methods}

Antibodies. Monoclonal antibodies to M1 and C1 antigens have been described and partly characterized (Lagenaur et al., 1980; Sommer et al., 1981). M1 arose from a rat immunized with cerebellar particulate fraction; $\mathrm{C} 1$ arose from a mouse immunized with bovine corpus callosum. Supernatants of hybridoma cells (either M1 or C1) were concentrated by precipitation with $50 \%$ satu- 
rated ammonium sulfate. For immunohistology, these antibodies were used at a concentration that was 2 -fold greater than the original hybridoma supernatant. Antisera to glial fibrillary acidic (GFA) protein were generous gifts from Drs. A. Bignami, D. Dahl, and L. F. Eng. Fluorescein isothiocyanate (FITC)-conjugated goat antimouse immunoglobulins were purchased from Antibodies, Inc. (Paesel, Frankfurt, FRG) and were used at a dilution of 1:50. Sheep anti-rat immunoglobulins were labeled with FITC, purified by affinity chromatography as previously described (Lagenaur and Schachner, 1981), and used at a dilution of 1:50. Tetramethylrhodamineconjugated goat anti-rabbit IgG was purchased from Nordic Immunology (Byk Mallinckrodt, DietzenbachSteinberg, FRG) and used at a dilution of 1:300. Horseradish peroxidase-coupled rabbit anti-rat IgG was purchased from Miles (Frankfurt, FRG) and used at a dilution of 1:30.

Localized stab lesion to cerebellum. C57BL/6J mice (4 to 6 weeks old) were anesthetized with ether, and an incision was made through the skin to expose the posterior fossa. With a hand-held, motor-driven drill, a 1-mm bore hole was made through the calvaria to the right of the midline into the superior aspect of the cerebellum to a depth of 2 to $3 \mathrm{~mm}$. This procedure took less than 30 sec to perform, and the mice were allowed to recover, most without discernible ill effects or apparent neurological impairment. These injured mice, along with uninjured control mice, were sacrificed by cervical dislocation at intervals of $2,4,6,8,10,16,24,31$, and 50 days after injury. The brains were removed quickly and fresh frozen for immunohistological procedures. In cryostat sections of these brains (see below), the area of injury was macroscopically visible as an area of brownish discoloration affecting only a few of the superior cerebellar folia. Frozen sections of this area also were stained with toluidine blue for light microscopic examination of the injured area.

Immunohistological procedures. Indirect immunofluorescence microscopy of cryostat sections of fresh frozen brain was carried out as previously described (Goridis et al., 1978). In brief, the fresh frozen brains of control and injured animals were cut in the sagittal plane as $9-\mu \mathrm{m}$-thick cryostat sections, dried onto coverslips, and incubated at room temperature in a moist chamber with simultaneously applied monoclonal antibody and antiserum to GFA protein. The sections were washed

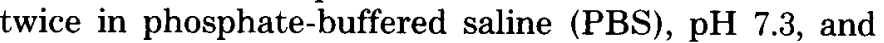
incubated, again simultaneously, with the fluorochromeconjugated antibodies to rabbit and mouse or rat immunoglobulins. Sections again were washed twice in PBS and examined with a Zeiss fluorescence microscope with epi-illumination and appropriate filters. Adjacent serial sections were used for immunolabeling with $\mathrm{M} 1$ and $\mathrm{C} 1$ antibodies. The peroxidase labeling was developed for 10 min with a solution containing $0.5 \mathrm{mg} / \mathrm{ml}$ of 4 -chloro-1naphthol (Sigma, Munich, FRG), 15\% methanol, $0.15 \mathrm{M}$ $\mathrm{NaCl}$, and $50 \mathrm{~mm}$ Tris, $\mathrm{pH} 7.2$.

\section{Results}

Light microscopic examination of the stab lesion in toluidine blue-stained frozen sections of adult mouse cerebellum showed that, 2 days after injury, there was a localized inflammatory cell response surrounding the area of tissue disruption. From days 4 to 10, the inflammatory response decreased in the area of injury with the appearance of increased numbers of large nuclei in each layer of the cerebellum, possibly of astrocytic origin. By day 24, no inflammatory cells were seen. Fibrosis was seen in meninges overlying the injury with the accumulation of hemosiderin-like matcrial, and there was an increase in astrocytic nuclei in the area of the wound. At days 31 and 50, the lesion was not detectable macroscopically. Sections through the presumptive area of injury showed a mild meningeal fibrosis. At each stage, sections were stained by indirect immunofluorescence with M1 and $\mathrm{C} 1$ antibodies. These experiments are described below and summarized in Table I. Examples of the distribution of $\mathrm{M} 1$ and $\mathrm{C} 1$ antigens in normal adult cerebellum are shown in Figure 1.

TABLE I

Expression of $M 1$ and $C 1$ antigen in adult mouse cerebellum as a function of time after infliction of a stab wound

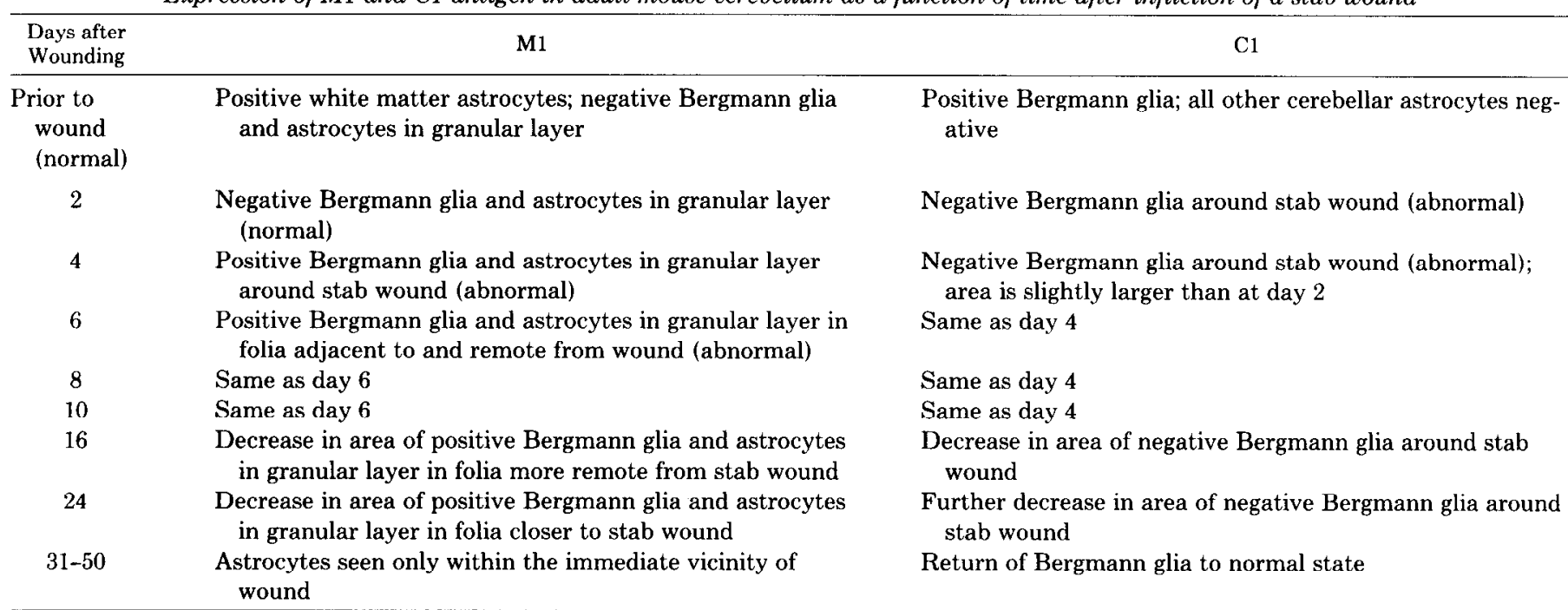



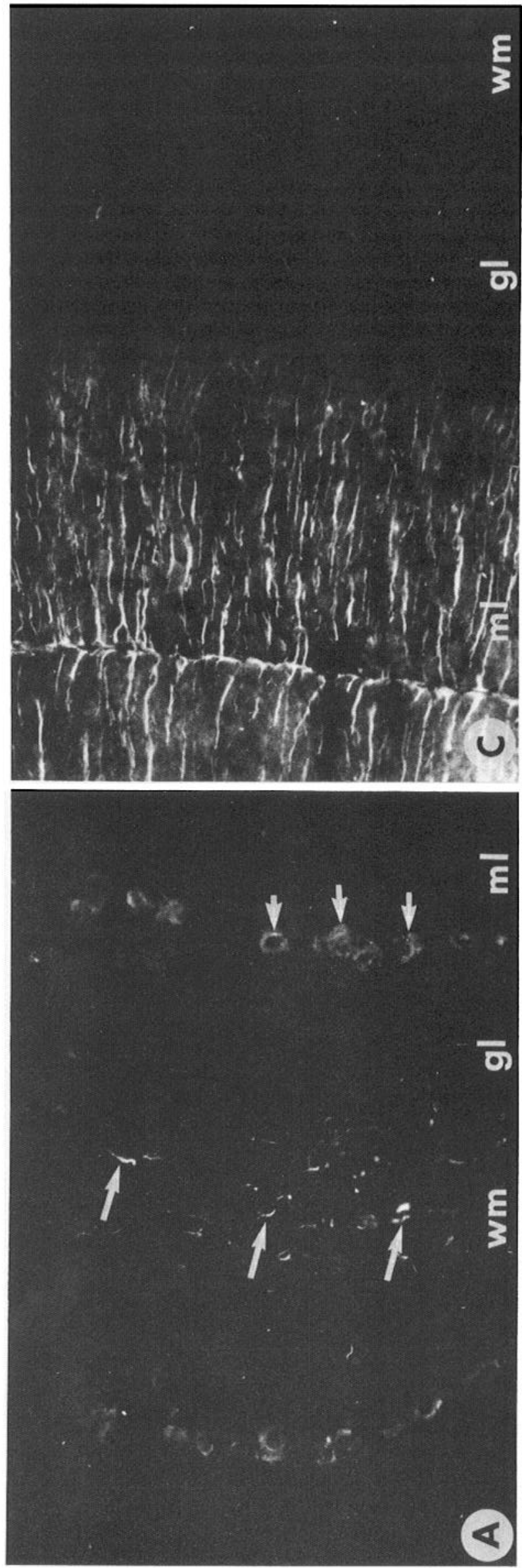

4
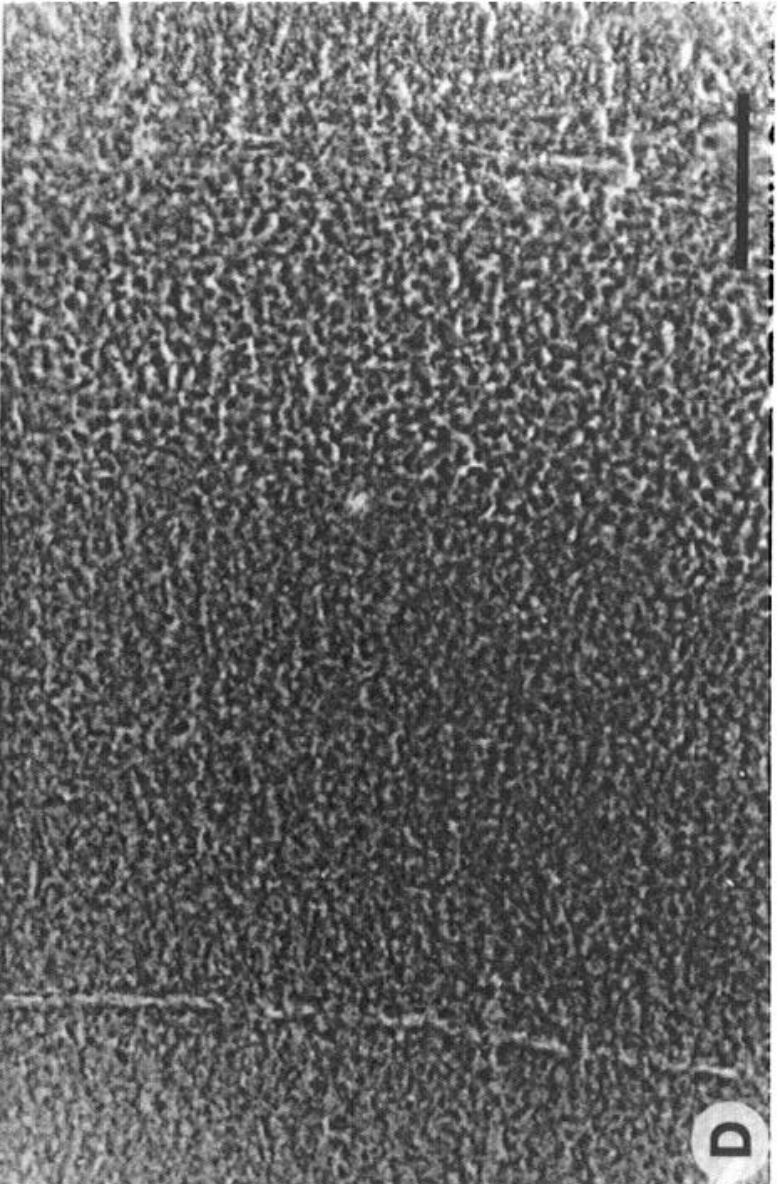

8

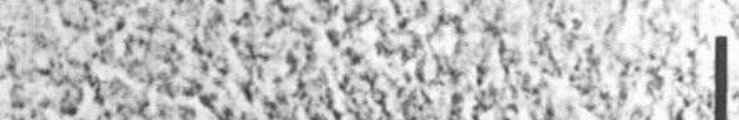

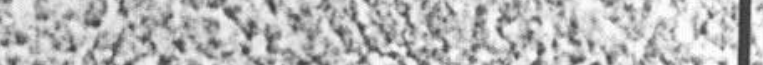

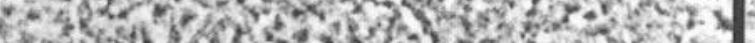

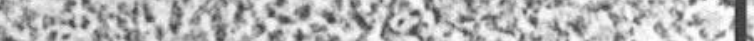

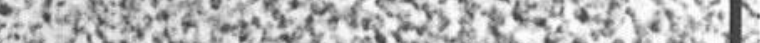

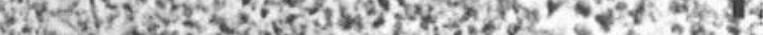

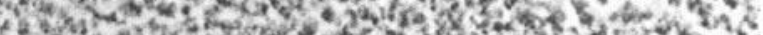

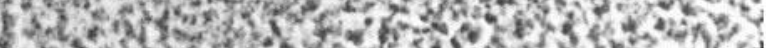

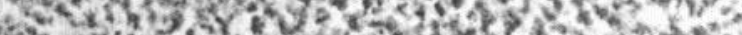

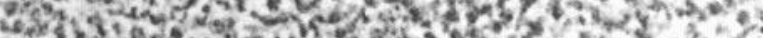

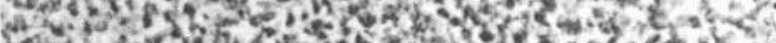

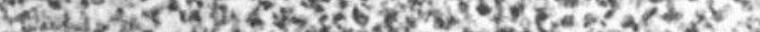

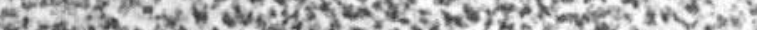

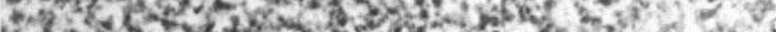

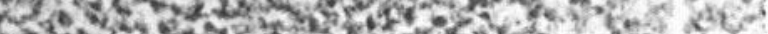
Wh 3hy

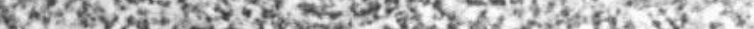

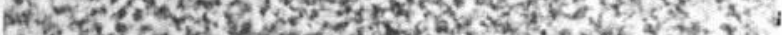

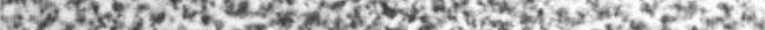

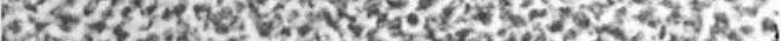

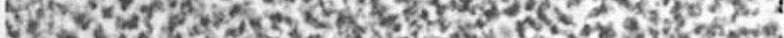

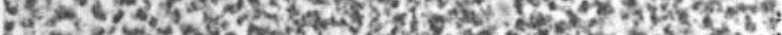

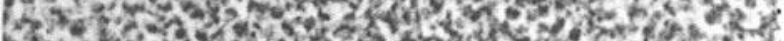

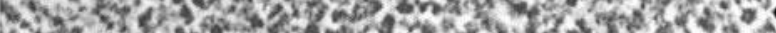

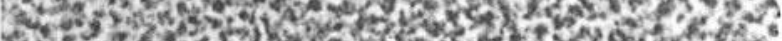

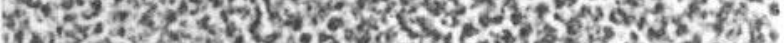

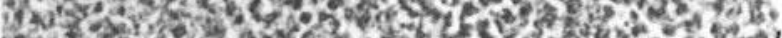

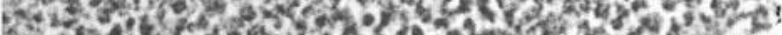

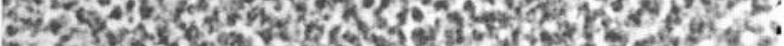

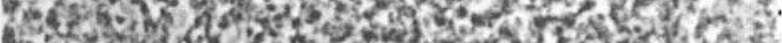

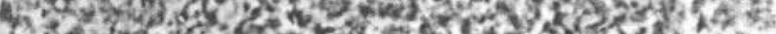

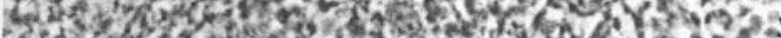

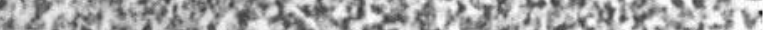

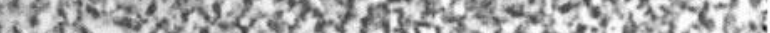

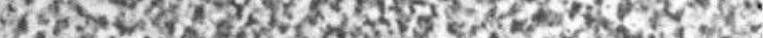

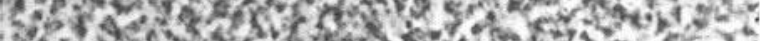
3620.720 .

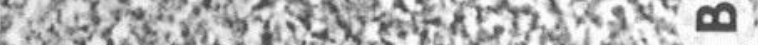

串范荡

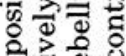

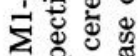

क्षे क्ष

ชิธี

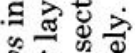

ริ สี

송

응 范

ปู่

․ㅀ

हี่

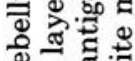

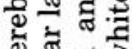

ญ

든

ㄷํ 50

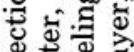

㐘

สำ 모

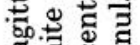

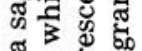

झ

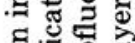

해윯

.

สี ฉี हี

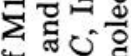

प๐

$\infty$ का

등유.

용

웡

论

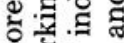

댕 डิ के छี

릻요

हี कै के 눈등 웡

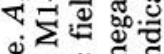

¿.

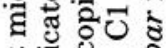

플 司芯

के क्ड

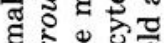

ชิ สี

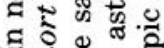

ఏ 웡

牙宁至

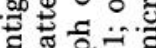

৩.

즐. 음

론

$\sum$ 范范

군

ऽ

웡 है

工 
M1 antigen. M1 antigen was clearly detectable by indirect immunofluorescence at day 4 after injury in astrocytes which normally (in uninjured control brains) do not express the antigen: Bergmann glia and astrocytes of the granular layer (Figs. 2, 3, and 4). M1 antigen was first seen in these astrocytes in the immediate vicinity of the wound. As shown in Figure 2, the astrocytes that abnormally express M1 antigen are confined initially to the folia adjacent to the wound. Figures 3 and 4 show greater detail of a typical wound area and adjacent folia, respectively, and demonstrate the location of GFA protein-bearing astrocytes. By double immunolabeling using antiserum to GFA protein, astrocytes in all layers of the cerebellum could be seen, including those hypertrophied astrocytes present within and surrounding the wound at day 1 after injury. The fact that GFA protein-positive, but M1-negative glia could be readily demonstrated showed that penetration of antibodies is not a limiting factor in detectability of M1 antigen. Furthermore, GFA protein served as a marker to unequivocally identify the M1 antigen-positive cells as astrocytes (Figs. 3 and 4). At days 6,8 , and 10 after injury, M1 antigen was detectable in folia more remote from the site of injury, first within hypertrophied astrocytes of the granular layer and then in the Bergmann glia. A gradient of astrocytic response extended away from the area of injury. Outside of the actual wound area, M1 was detectable in astrocytes without apparent hypertrophy (see, for example, Figs. 2 and 4). From days 16 to 50 after injury, the M1 antigen disappeared from astrocytes in the granular and molecular layers in areas more remote from the lesion site, first disappearing from astrocytes of the molecular and then from those of the granular layer. Even at 50 days after injury, M1-positive Bergmann glia were seen within and surrounding the area of injury.

Purkinje cells which have been found to express M1 antigen in adult mice (Lagenaur et al., 1980) show a decreased level or absence of M1 antigen within the area of injury between days 4 and 24 (Fig. 3). Due to the disruption of normal cerebellar morphology in the area of the wound, it is difficult to determine if some or all loss of M1 antigen from Purkinje cells may be due to the death of these cells.

C1 antigen. Two days after injury, $\mathrm{C} 1$ antigen was no longer detectable in the immediate vicinity of the injury within Bergmann glia which normally express $\mathrm{C} 1$ antigen. Similar to the localized area of injury which showed abnormal expression of M1 antigen by day 4, the loss of C1 antigen was restricted to those folia closest to the wound at day 2 . By day 4 after injury, the area containing $\mathrm{C} 1$ antigen-negative Bergmann glia enlarged to encompass the folia adjacent to the wound (Fig. 5). At none of the intervals following injury did $\mathrm{C} 1$ antigen appear in GFA protein-positive astrocytes in the granular layer or white matter, neither within nor remote from the lesion. Endothelial cells, as demonstrated by a marker for the glycoprotein fibronectin (Schachner et al., 1978), showed detectable levels of $\mathrm{C} 1$ antigen in the center of the wound from days 4 to 10 .

At days 6,8 , and 10 , the size of the $\mathrm{C} 1$ antigen-negative area was similar to that seen at day 4 ; thus C1 loss was more restricted, relative to the widespread abnormal M1 expression. From days 16 to 24 , the area containing $\mathrm{C} 1$ antigen-negative Bergmann glia decreased, and by days

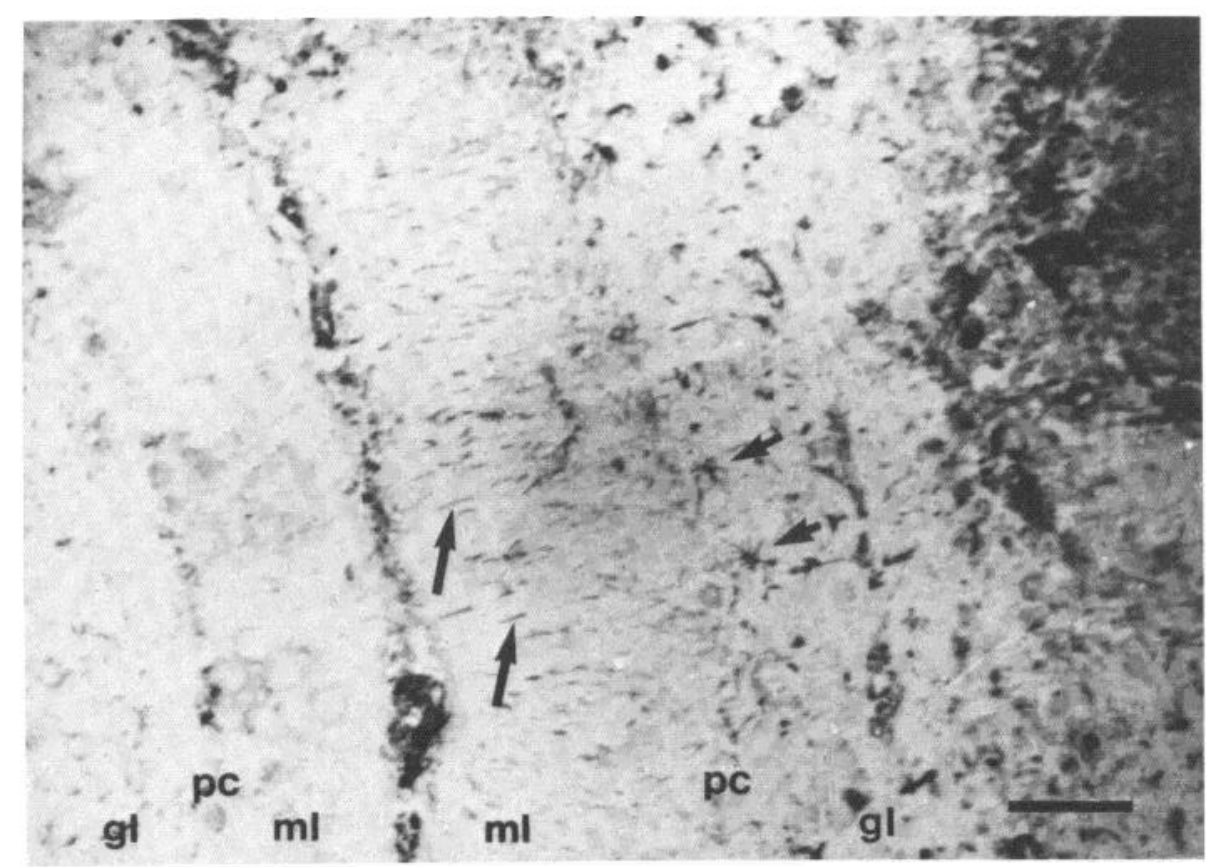

Figure 2. Indirect peroxidase staining of abnormal M1 expression 4 days after injury. The wound is visible in the upper right corner of this microscopic field (darkened area). Long arrows indicate M1-positive Bergmann glia in the same folia; short arrows indicate hypertrophied granular layer astrocytes. Note that, in the folia to the left, no M1 is detectable in Bergmann glia. gl, $p c$, and $m l$ indicate granular layer, Purkinje cell layer, and molecular layer, respectively; bar indicates $100 \mu \mathrm{m}$. 

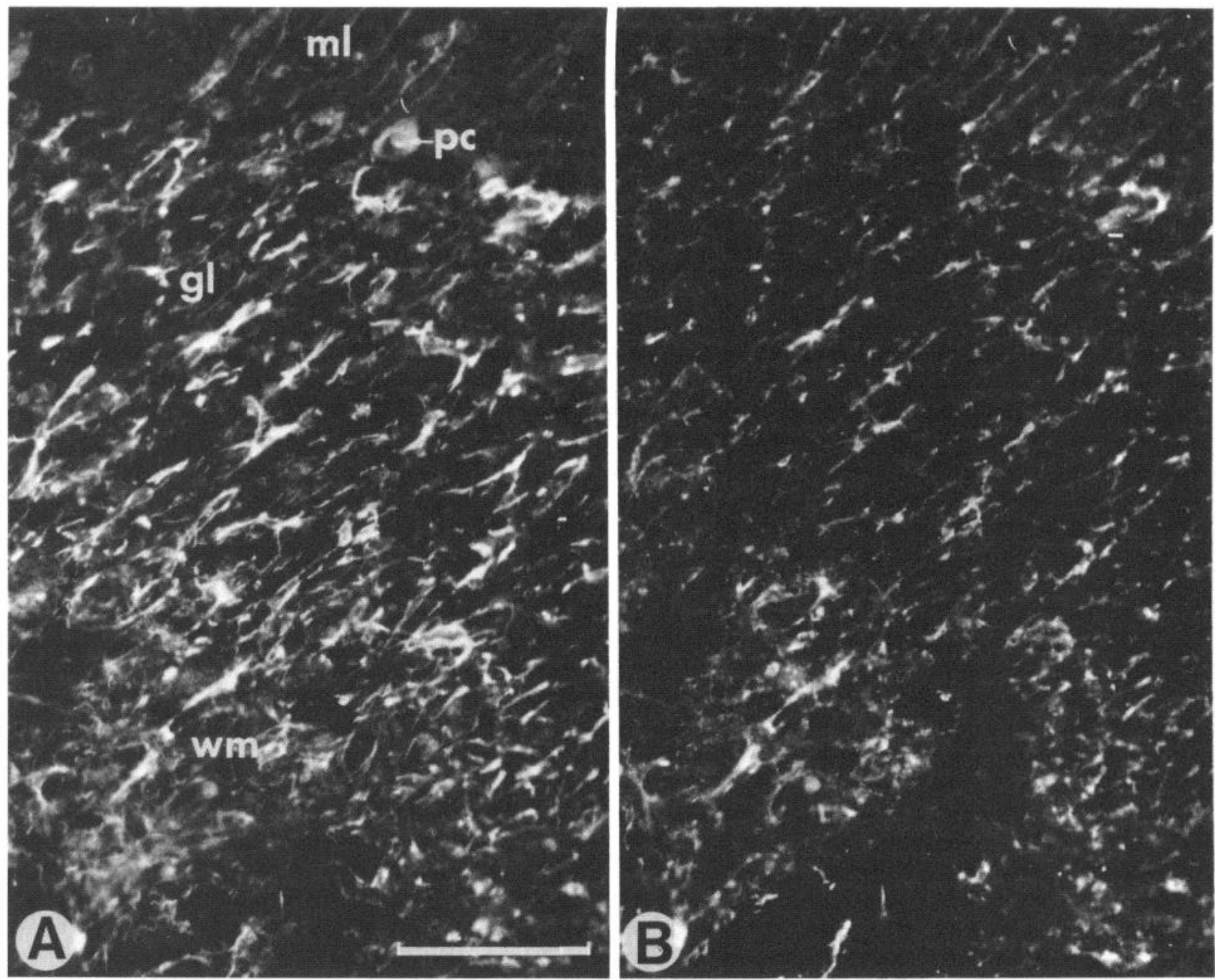

Figure 3. Double immunofluorescence staining of M1 and GFA protein at the site of the stab lesion 3 days after injury. Hypertrophied astrocytes, typical of a reactive glial response to wounding, are seen at and surrounding the site of the lesion (bottom of figure). A, M1 antigen is detected in astrocytes of white matter $(\mathrm{wm})$, granular layer $(\mathrm{gl})$, and molecular layer $(\mathrm{ml})$ as well as in Purkinje cells $(p c)$. The reaction in Purkinje cells is weaker than in the normal cerebellum. $B$, GFA protein identifies astrocytes in the same microscopic field as shown in A. Bar indicates $100 \mu \mathrm{m}$.

31 and 50, practically all GFA protein-positive Bergmann glia had re-expressed $\mathrm{C} 1$ antigen in the lesion.

\section{Discussion}

The present study has shown that M1 and C1 antigens not only distinguish among subsets of astroglia in the normal adult mouse cerebellar cortex but also that these antigens are sensitive indicators of a cellular response to a pathological situation: specifically, the glial response resulting from injury inflicted by stab wounding. As a result of this mechanical lesion in the cerebellum, M1 is expressed abnormally in Bergmann glia and in astrocytes of the granular layer. Concomitant with the abnormal rise in expression of $\mathrm{M} 1$, expression of $\mathrm{C} 1$ antigen in Bergmann glia subsides in the vicinity of the wound.

Reactive gliosis has been described to result in an increased expression of glial fibrillary acidic (GFA) protein in affected astrocytes (Bignami and Dahl, 1976; Latov et al., 1979). Amaducci et al. (1981) observed enlarged astrocytes which immunolabeled intensely for GFA protein in the vicinity of a cold-induced lesion as early as $30 \mathrm{~min}$ after the lesion and lasted for at least 2 days after the lesion. In our studies, which examined glial reactions during a period of 2 to 50 days after injury, GFA protein also was observed to be strongly expressed in hypertrophied astrocytes in the immediate vicinity of the wound.

The maximal expression of M1 in astrocytes in response to stab wounding occurred 8 to 12 days after the injury. At this time, abnormal M1 expression extended over most of the cerebellar hemisphere, affecting regions that showed no hypertrophy of astrocytes. Thus, M1 expression may be a more subtle indicator of a reactive response in astrocytes than hypertrophy or increased expression of GFA protein. C1 antigen disappeared from Bergmann glia in a more restricted area around the lesion, whereas abnormal M1 antigen expression was considerably less confined.

The observation that the expression of M1 and C1 antigens is modulated during normal development, in normal cerebellar astrocytic subclasses, now can be considered in light of the response of these antigens to injury. M1 expression is most prominent in all types of cerebellar 

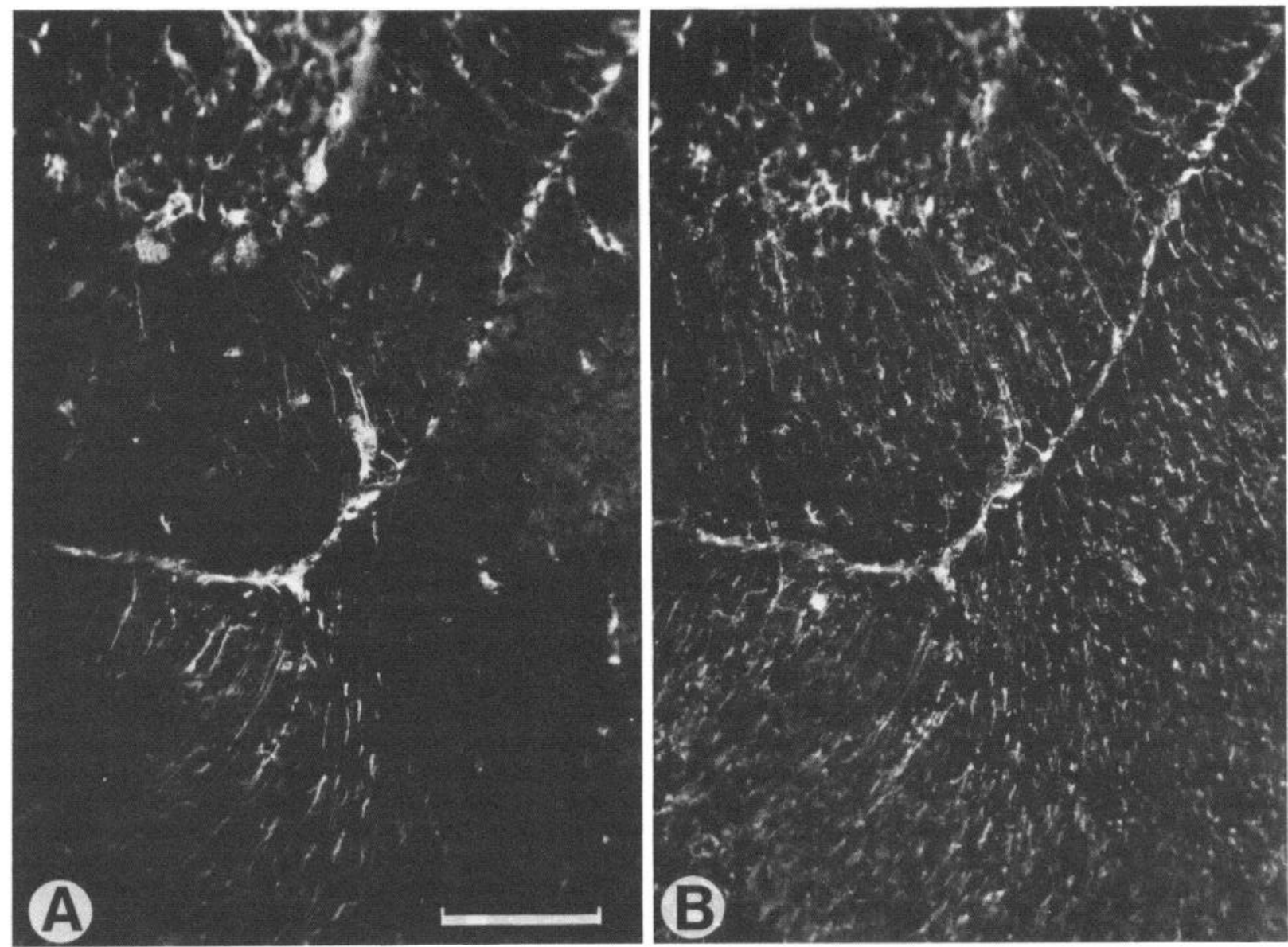

Figure 4. Double immunofluorescence staining of M1 and GFA protein at the margin of detectable abnormal M1 expression in cerebellum 4 days after injury. In the field shown, Bergmann glia with apparently normal morphology are observed to express M1 antigen several folia remote from the lesion site, which was below and to the left of the microscopic field shown here. $A$, M1 antigen; $B$, GFA protein identifies astrocytes in the same microscopic field as shown in $A$. Bar indicates $100 \mu \mathrm{m}$.

astrocytes, including Bergmann glia, when gliogenesis and myelination are at their peak between the end of the 1st and beginning of the 3rd postnatal week (Lagenaur et al., 1980). At the same time, C1 antigen becomes hardly detectable in astrocytes in white matter and the granular layer and in Bergmann glia, reappearing in Bergmann glia only after the 3rd postnatal week (Sommer and Schachner, 1981). These combined observations from normal development and the pathological response seen in this study support a model in which M1 antigen expression and $\mathrm{C} 1$ antigen repression would represent a reactive and/or proliferative state of astrocytes. The actual contribution of glial proliferation to reactive gliosis is unknown; however the results of Latov et al. (1979) do demonstrate increased levels of astrocyte division in response to central nervous system wounding. The results presented here would suggest that there is a gradient of reactive glial response. Within the wound site, astroglial hypertrophy as well as abnormal M1 expression are observed, while at more distant sites, only abnormal M1 expression is detected. It remains unclear whether those astrocytes that normally express M1 antigen in adult brain (e.g., those in cerebellar white matter) represent a more "reactive" state compared to M1-negative astrocytes in the granular and molecular layers. Although quantitative measurements are very difficult using the immunofluorescence technique, white matter astrocytes near the wound site consistently appeared to have increased levels of M1 relative to unwounded areas. $\mathrm{C} 1$, in contrast, appears at very early stages of glial development and is retained only in a particular class of astroglia which are direct descendants of the primitive radial glia, such as the Bergmann glia and the retinal Müller cells (Sommer et al., 1981). Thus, C1 antigen expression might be characteristic of a more ontogenetically primitive and "unreactive" state of astrocytes.

In agreement with these hypotheses are the observations that, in four neurological mutations of the mouse (weaver, staggerer, reeler, and Purkinje cell degeneration), M1 antigen is expressed and $\mathrm{C} 1$ antigen is repressed in Bergmann glia (Sommer and Schachner, 1981), in which reactive gliosis might occur in response to neuronal cell death and/or abnormal environment. At stages when the abnormal mutant phenotypes are fully apparent, M1 antigen is detectable in Bergmann glia and astrocytes of the granular layer, while $\mathrm{C} 1$ antigen is no longer present in Bergmann glia throughout the mediolateral and dorsoventral aspects of the cerebellum (Lagenaur et al., 1980; Sommer et al., 1981; Sommer and Schachner, 1981). Since the timing of the M1 and C1 changes seen in the various mutants is related to the order of appearance of the morphologically recognizable mutant phenotypes, 

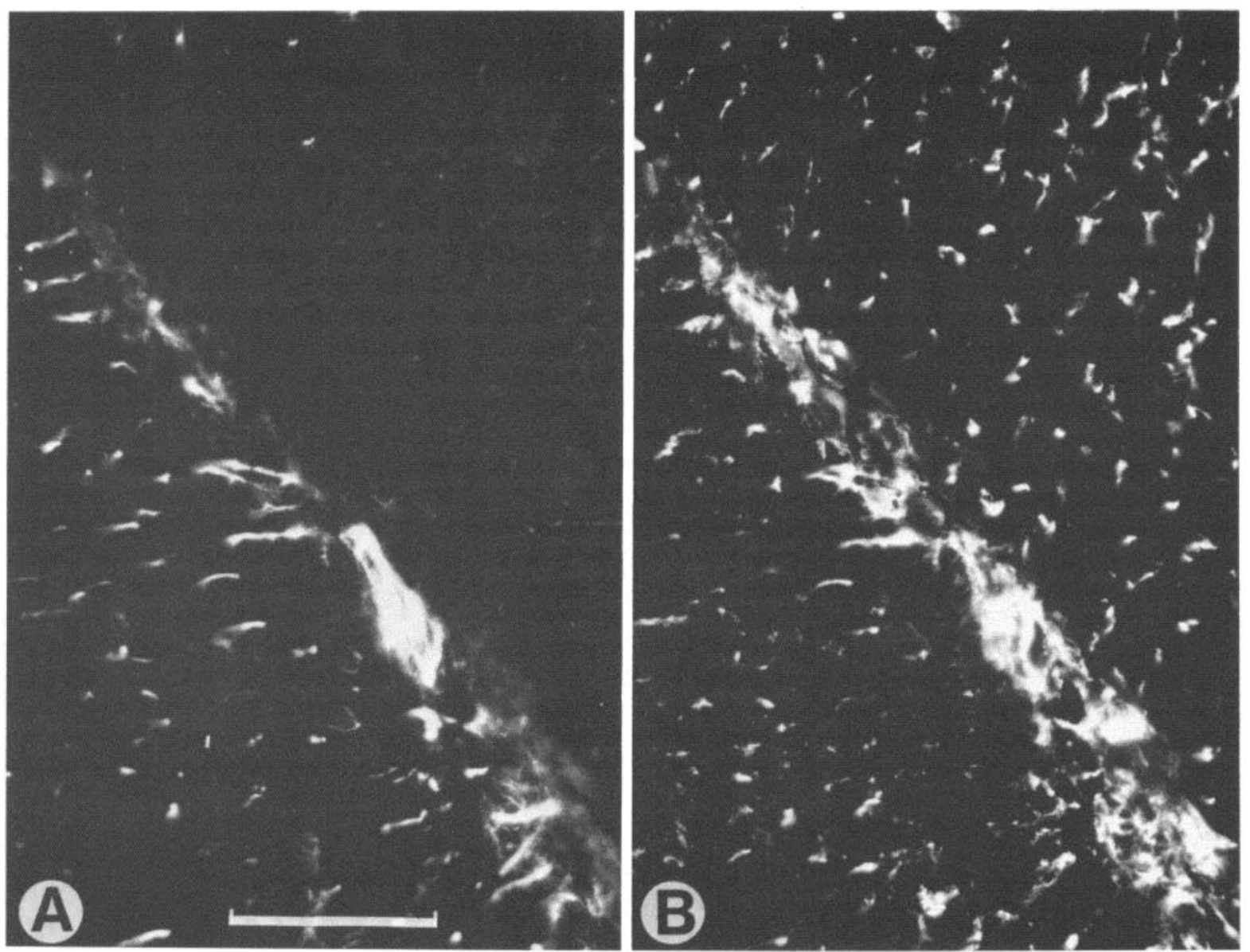

Figure 5. Double immunofluorescence staining of $\mathrm{C} 1$ and GFA protein at the margin of a lesion showing the Ioss of detectability of $\mathrm{C} 1$ antigen from cerebellar Bergmann glia in the vicinity of the lesion 4 days after injury. This figure shows the boundary between two folia; the wound area extended into the folia shown in the upper right. $A, \mathrm{C1}$ antigen; $B$, GFA protein identifies astrocytes in the same microscopic field as shown in A. Bar indicates $50 \mu \mathrm{m}$.

these markers may be useful in defining the time at which these mutants first exhibit reactive gliosis. It also will be interesting to see if other brain areas of these mutants show abnormal M1 or C1 expression.

The molecular mechanisms underlying the expression of M1 and C1 antigens are presently unknown, and elucidation of the signals involved will have to await the characterization of the antigens' molecular nature.

\section{References}

Amaducci, L., L. I. Forno, and L. F. Eng (1981) Glial fibrillary acidic (GFA) protein in cryogenic lesions of the rat brain. Neurosci. Lett. 21: 21-32.

Bignami, A., and D. Dahl (1976) The astrocyte response to stabbing: Immunofluorescence studies with antibodies to astrocyte-specific protein (GFA) in mammalian and submammalian vertebrates. Neuropathol. Appl. Neurobiol. 2: 99-110. Goridis, C., J. Martin, and M. Schachner (1978) Characteriza- tion of an antiserum to synaptic glomeruli from rat cerebellum. Brain Res. Bull. 3: 45-52.

Lagenaur, C., and M. Schachner (1981) Monoclonal antibody M2 to glial and neuronal cell surfaces. J. Supramol. Struct. 15: $335-346$.

Lagenaur, C., I. Sommer, and M. Schachner (1980) Subclass of astroglia in mouse cerebella recognized by monoclonal antibody. Dev. Biol. 79: 367-378.

Latov, N., G. Nilaver, E. A. Zimmerman, W. G. Johnson, A. J. Silverman, R. Defendini, and L. Cote (1979) Fibrillary astrocytes proliferate in response to brain injury. Dev. Biol. 72: 381-384.

Schachner, M., G. Schoonmaker, and R. O. Hynes (1978) Cellular and subcellular localization of LETS protein in the nervous system. Brain Res. 158: 149-158.

Sommer, I., and M. Schachner (1981) Expression of glial antigens $\mathrm{C} 1$ and $\mathrm{M} 1$ in developing and adult neurologically mutant mice. J. Supramol. Struct. 16: 53-74.

Sommer, I., C. Lagenaur, and M. Schachner (1981) Recognition of Bergmann glial and ependymal cells in the mouse nervous system by monoclonal antibody. J. Cell Biol. 90: 448-458. 